Un qualunque sistema di forme lineari $\sum_{j=1}^{n !} a_{i j} \pi_{j}(i=1,2, \ldots)$ a coefficienti interi razionali definise un tipo di simmetria nel modulo delle forme differen. ziali di grado $n$, se si stabilisee che l'appartenere di $x \in K \cdot(d K)^{n}$ a quel tipo significhi il soddisfare alle equazioni

$$
\sum_{j=1}^{n !} a_{i j} \pi_{j} x=0 \quad(i=1,2, \ldots)
$$

Rileviamo come il più importante tipo di simmetria l'antisimmetria definita dalle relazioni $x-\chi\left(\pi_{i}\right) \cdot \pi_{i} x=0 \quad(i=1,2, \ldots, n !)$.

\title{
§ 3. Differenziali integri di un corpo.
}

351. Sia $B$ un insieme, finito o no, di elementi del corpo $K(\supset k)$, i cui differenziali generici (sopra $k$ ) $d_{1} x$ costituiscano una base linearmente indipendente di $K \cdot d_{1} K=\sum_{x \in B} K \cdot d_{1} x$. Vale allora anche $K \cdot d_{i} K=\sum_{x \in B} K \cdot d_{i} x$ * l'indipendenza lineare dei $d_{i} x(x \in B)$ per ogni altro indice $i$. e la corrispondenza infinitesimale $R=\left[K, d_{1} K, d_{2} K, \ldots\right]$ di $K$ (relativa a $k$ ) sarà

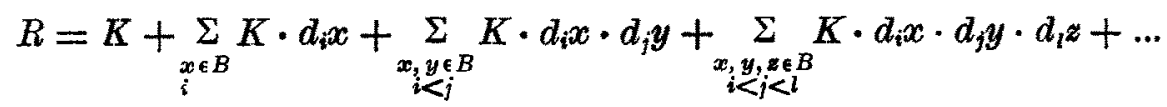

Considerando per ora gli elementi $d_{i} x(i=1,2,3, \ldots, x \in B)$ come argomenti liberi di un anello $P$ di polinomi a coefficienti in $K$, potremo dire che $R$ risulta da $P$ mediante un omomorfismo avente il nucleo

$$
\sum_{x, y \mathrm{e} B} d_{i} x \cdot d_{i} y \cdot P
$$

(ved. 4 e 5) e che pertanto ogni elemento di $R$ è uguale a una sola espressione del tipo indicato al membro destro di (*).

352. La corrispondenza differenziale $S$ di $K$ (ved. 9) si deriva da $R$ per mezzo di un omomorfismo avente il nucleo

$$
\mathrm{n}=\sum_{x, y \in B}\left(d_{i} x \cdot d_{j} y+d_{i} y \cdot d_{j} x\right) \cdot R,
$$

al quale corrisponde in $P$ l'ideale

$$
\sum_{x, y \in B} d_{i} x \cdot d_{i} y \cdot P+\sum_{x, y \in B}\left(d_{i} x \cdot d_{j} y+d_{i} y \cdot d_{j} x\right) \cdot P
$$

Calcolare in $K$ modulo $\mathrm{n}$, equivale a introdurre l'antisimmetria espressa dalle congruenze

$$
d_{i} x \cdot d_{j} y+d_{i} y \cdot d_{j} x \equiv 0 \quad(x, y \in B)
$$


le quali nel caso di car $K \neq 2$ permettono di ridurre ogni elemento di $R$ alla forma

$$
a+\sum_{n \geq 1} \underset{i_{1}<i_{2}<\ldots<i_{n}}{\Sigma} \underset{i_{1}<j_{2}<\ldots<j_{n}}{\Sigma} a_{i_{1} i_{2} \ldots i_{n}}^{j_{1} j_{2} \ldots j_{n}} \cdot d_{i_{1}} x_{j_{1}} \cdot d_{i_{2}} x_{j_{2}} \cdot d_{i_{n}} x_{i_{n}},\left(x_{j} \in B\right) .
$$

Nel caso di car $K=2$, si avrà simile forma ridotta che si distingue dalla suddetta solamente con ciò che si deve ammettere ugualità fra gli indici $j_{1}, \ldots, j_{n}$.

Dimostreremo ora nel caso di car $K \neq 2$, che una espressione ridotta del tipo (*) non rappresenta un elemento di $\mathfrak{n}$, a meno che siano 0 tutti $i$ suoi coefficienti $a, a_{i_{1} \ldots i_{n}}^{j_{1} \ldots j_{n}}$.

Data la liberta degli argomenti $d_{i} x$ in $P$, l'ipotesi (*) $\subset \mathfrak{n}$ si traduce in un sistema di equazioni valide in $P$ :

$$
\begin{gathered}
\Sigma^{\prime} a_{i_{1} \ldots i_{n}}^{j_{1} \ldots j_{n}} \cdot d_{i_{1} x_{j_{1}} \ldots d_{i_{n}} x_{j_{n}}}=\sum_{h, j}\left(d_{h_{1}} x_{j_{1}} \cdot d_{h_{2}} x_{j_{2}}+d_{h_{1}} x_{j_{2}} \cdot d_{\left.h_{2} x_{j_{1}}\right)} \cdot\right. \\
\cdot b_{i_{1} \ldots i_{n}}^{j_{1} \ldots j_{n} h_{1} \ldots h_{n}} \cdot d_{h_{3} x_{j_{3}} \ldots d_{h_{n}} x_{j_{n}}}
\end{gathered}
$$

dove il segno $\Sigma^{\prime}$ indichi sommazione con ristrezione a $j_{1}<j_{2}<\ldots<j_{n}$. Gli indici $h_{1}, \ldots, h_{n}$ percorrono le permutazioni del sistema $i_{1}, \ldots, i_{n}$ supposto fisso.

Possiamo limitarci alla considerazione dei termini al membro destro di $(* *)$, dove $h_{1}, \ldots, h_{n}$, e $j_{1}, \ldots, j_{n}$ sono permutazioni $\sigma(1), \ldots, \sigma(n)$, risp. $\tau(1), \ldots, \tau(n)$ di $1, \ldots, n$.

Scrivendo $(\sigma, \tau)$ invece di

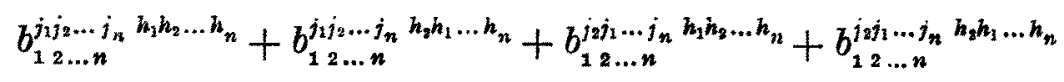

sicchè sarà $(\sigma \rho, \tau)=(\sigma, \tau \rho)=(\sigma, \tau)$, se $\rho$ è la trasposizione che cambia 1 in 2, troveremo

$$
\sum_{\sigma} \sum_{i=1}^{N}\left(\sigma \tau_{i}, \tau_{i}\right) \cdot d_{1} x_{\sigma(1)} \cdot d_{2} x_{\sigma(2)} \ldots d_{n} x_{\sigma(n)}
$$

( $\tau_{i}$ percorre le $N=\frac{n !}{2}$ permutazioni pari) come l'insieme dei termini del membro destro in $(* *)$ formati solamente con $d_{i} x_{j}(i, j \leq n)$. Siccome al mem. bro sinistro non intervengono termini det tipo $a \cdot d_{1} x_{\sigma(1)} \cdot d_{2} x_{\sigma(2)} \ldots d_{n} x_{\sigma(n)}$ con $\sigma \neq 1$, debbono sussistere le relazioni

mentre

$$
\sum_{i=1}^{N}\left(\sigma \tau_{i}, \tau_{i}\right)=0 \quad(\sigma \neq 1),
$$

$$
a_{1 \ldots n}^{1 \ldots n}=\sum_{i=1}^{N}\left(\tau_{i}, \tau_{i}\right)
$$


Nell' anello $R=\underset{\sigma \varepsilon G}{\Sigma}[1] \sigma$ del gruppo $G$ delle $n !$ permutazioni sia

$$
\sigma \tau_{i}=\left\{\begin{array}{l}
\mathrm{\Sigma} a_{i j}(\sigma) \tau_{j}, \text { se } \chi(\sigma)=1, \text { cioè } \sigma \text { è pari, } \\
\mathrm{\Sigma} a_{i j}(\sigma) \tau_{j}, \text { se } \chi(\sigma)=-1, \text { ciò̀ } \sigma \text { è dispari. }
\end{array}\right.
$$

Le matrici $A(\sigma)=\left(a_{i j}(\sigma)\right)$ che rappresentano il gruppo $G$ nel senso $A\left(\sigma^{\prime} \sigma\right)=$ $=A(\sigma) A\left(\sigma^{\prime}\right)$, soddisfano allora alla relazione

$$
\sum_{\sigma \varepsilon G} \chi(\sigma) A(\sigma)=0
$$

Invero, se la matrice $B$ al membro sinistro non fosse 0 , si otterrebbe, formando

$$
B \cdot\left(\begin{array}{c}
\tau_{1} \\
\vdots \\
\tau_{N}
\end{array}\right)=\left(\begin{array}{c}
L_{1} \\
\vdots \\
L_{N}
\end{array}\right)
$$

almeno una forma lineare $L=L_{v}=\sum_{i=1}^{N} \lambda_{i} \tau_{i} \neq 0$, la quale di seguito a $B \cdot A(\sigma)=X(\sigma) B$ arrebbe le proprietà

$$
\begin{array}{ccc}
\sigma L=L & \text { per } & \chi(\sigma)=1 \\
\sigma L=-L \rho & \text { per } & \chi(\sigma)=-1 .
\end{array}
$$

Ora dnlla prima segue, prendendo $\sigma=\tau_{i}(i=1, \ldots, N)$ che tutti $i$ coefficienti $\lambda_{i}$ debbono essere uguali fra di loro, sicchè $\rho L=-L \rho$ contraddice al fatto $p L=p\left(\underset{i}{\Sigma} \lambda \tau_{i}\right)=\sum_{i} \lambda \tau_{i} 0=L p$, se non $L=0$.

Dimostrate le relazioni

$$
\underset{\sigma \varepsilon G}{\Sigma} \chi(\sigma) a_{i j}(\sigma)=0 \quad(i, j \leq N)
$$

equivalenti a $(+t)$, ne ricaviamo

$$
\underset{\sigma \sigma \varepsilon G}{\Sigma} \chi(\sigma) \sum_{i=1}^{N}\left(\sigma \tau_{i}, \tau_{i}\right)=\underset{\sigma}{\Sigma} \chi(\sigma) \underset{i, i}{\sum} a_{i_{j}}(\sigma)\left(\tau_{j}, \tau_{i}\right)=0
$$

il the mostra che dal sussistere di $(+)$ consegue

c. d. d.

$$
\sum_{i=1}^{N}\left(\tau_{i}, \tau_{i}\right)=0, \text { ciò̀ } a_{1 \ldots n}^{1 \ldots n}=0,
$$

353. Ogni espressione

$$
d x \wedge d y+d y \wedge d x=d_{1} x \cdot d_{2} y+d_{1} y \cdot d_{2} x \quad(x, y \in K)
$$

e ogni prodotto esterno $X \wedge[d x \wedge d y+d y \wedge d x] \wedge Y$ con $X, Y \in M$ sono elementi dell'ideale $\mathfrak{n}$. Ne segue che l'ideale bilaterale

$$
\mathrm{\partial}=\sum_{x, y \in K} M \wedge[d x \wedge d y+d y \wedge d x] \wedge M
$$


generato dalle espressioni (*) nell' anello $M=[K, d K]=\sum_{n=0}^{\infty} K \cdot(d K)^{n}$ (ved. 349) è contenuto in $\mathfrak{n}$. Dimostreremo nel caso di car $K \neq 2$ che

$$
\mathfrak{c}=\mathfrak{n} \cap M .
$$

Sia

$\left(*_{* *}^{*}\right) \quad a+\sum_{n \geq 1} \sum_{i_{1} \ldots i_{n}} a_{i_{1} \ldots i_{n}} \cdot d x_{i_{1}} \wedge \ldots \wedge d x_{i_{n}} \quad\left(a, a_{i_{1} \ldots i_{n}} \in K\right)$

elemento qualunque di $\mathfrak{n} \frown M$, espresso mediante un sistema (finito) di elementi $x_{i}$ appartenenti aila base $B$ definita in $\mathbf{3 5 1}$.

In ogni termine $a_{i_{1} \ldots i_{r} i_{r+1} \ldots i_{n}} \cdot d x_{i_{1}} \wedge \ldots \wedge d x_{i_{n}} \wedge d x_{i_{r+1}} \wedge \ldots \wedge d x_{i_{n}} \mathrm{di}\left({ }_{* *}^{*}\right)$ si può invertire, con la sola addizione di un elemento di $\partial$, e cioè di

$$
-a_{i_{1} \ldots i_{r} i_{r+1} \ldots i_{n}} \cdot d x_{i_{1}} \wedge \ldots \wedge\left(d x_{i_{n}} \wedge d x_{i_{r+1}}+d x_{i_{r+1}} \wedge d x_{i_{r}}\right) \wedge \ldots \wedge d x_{i_{n}},
$$

l'ordine di due fattori consecutivi, cambiando però il segno del coefficiente. Siccome ogni permutazione lascia effettuarsi con una serie di permutazioni di fattori consecutivi, l'elemento $\left(\begin{array}{c}* \\ *\end{array}\right)$ è congruo mod $\partial$ a uno del tipo ridotto nel senso spiegato in 3.52. Dovendo questo appartenere a $n \cap M+\mathfrak{d} \subset \mathbf{n}$, esso e 0 , il che mostra $\left(*_{*}^{*}\right)$ essere elemento di 0 .

Da (**) segue $M+n / n \cong M / 0$ con l' isomorfismo che sostituisce $m+0$ a $m+\mathfrak{n}$ (per $m \in M$ ).

L'anello $M / \mathcal{O}$ e proprio quello che risulta dall' anello $M$, imponendogli la validità delle regole

$$
d x \wedge d y+d y \wedge d x \equiv 0 \quad(x, y \in K)
$$

Invero, l'ideale $\partial$ è costituito appunto colle espressioni atte a servire di membro sinistro in una relazione conseguente dalle $(+)$.

Se l'insieme $B$ è base nel senso spiegato in 351 e car $K \neq 2$, ogni ele. mento di $M / O$ può ridursi, con la sola applicazione delle regole $(+)$ ai differenziali base $d x(x \in B)$, a un' espressione del tipo

$$
a+\sum_{n \geq 1}^{\Sigma} \Sigma^{\prime} a_{i_{1} i_{2} \ldots i_{n}} \cdot d x_{i_{1}} \wedge d x_{i_{2}} \wedge \ldots \wedge d x_{i_{n}}
$$

dove $\Sigma^{\prime}$ designa sommazione sotto le condizioni $i_{1}<i_{2} \ldots<i_{n}$. Questa expres. pione ridotta è unica di seguito a $\partial \subset n$ (ved. 352).

Quell' isomorfismo $M+\mathfrak{n} / \mathfrak{M} M / \mathcal{O}$, riferentesi dapprima a $K$-moduli, ri. mane valido, qualora si considerino i suoi membri come anelli a moltiplica. zione esterna, postulando che

$$
(x+\mathfrak{n}) \wedge(y+n) \text { significhi } x \wedge y+n \text {, se } x, y \in M \text {. }
$$

Infatti, da $M \cap \mathfrak{n}=\mathrm{d}$ e $\mathrm{d} \wedge M \subset \mathrm{O}, M \wedge \partial \subset \mathrm{O}$ segue, che tanto $x \wedge y+\mathrm{n}$ quanto $x \wedge y+\mathrm{d}$ non dipendono dalla scelta di $x$ in $x+\mathfrak{n}$ e di $y$ in $y+n$.

Il fatto evidente, che l'ideale $\mathfrak{n}$ è invariante di fronte agli automorfismi $\pi$ indotti dalle permutazioni $\pi$, permette di interpretare tale $\pi$ anche come 
automorfismo di $R / \mathfrak{n}$, ponendo $\pi(x+\mathfrak{n})=\pi x+\mathfrak{n}$ (per $x \in R$ ). Al legame $R=\Sigma \pi M$ corrisponde quindi una simile relazione

$$
R / \mathfrak{n}=\Sigma \pi(M+\mathfrak{n} / \mathfrak{n})
$$

della corrispondenza differenziale al suo sotto- $K$-modalo $M+\mathfrak{n} / \mathfrak{n}$, la struttura del quale è ben conosciuta dalla sua interpretazione come anello isomorfo a $M / 0$.

304. La decomposizione di $R$ in $K$-moduli

$$
R=\sum_{n=0}^{\infty} \underset{i_{1}<\ldots<i_{n}}{\Sigma} K \cdot d_{i_{1}} \cdot K \ldots d_{i_{n}} K,
$$

diretta nel senso che da $\underset{n}{\Sigma} \underset{i_{1}<\ldots<i_{n}}{\Sigma} X_{i_{1} \ldots i_{n}}=0, \quad X_{i_{1} \ldots i_{n}} \in K \cdot d_{i_{1}} K \ldots d_{i_{n}} K$ segue $X_{i_{1} \ldots i_{n}}=0$, rimane diretta anche calcolando $\bmod \mathfrak{n}$. Infatti, questo ideale è somma di $K$-moduli

$$
\underset{x, y \in K}{\Sigma} \underset{h_{1} \ldots h_{n}}{\Sigma}\left(d_{h_{1}} x \cdot d_{h_{2}} y+d_{h_{1}} y \cdot d_{h_{2}} x\right) \cdot K \cdot d_{h_{3}} K \ldots d_{h_{n}} K \subset K \cdot d_{i_{1}} K \ldots d_{i_{n}} K \frown \mathfrak{n}
$$

$\left(h_{1} \ldots h_{n}\right.$ percorre le permutazioni di $\left.i_{1} \ldots i_{n}\right)$, del quale fatto ci siamo del resto gia serviti in $35 \%$.

Per poter effettuare $\mathrm{i}$ calcoli in $R / \mathfrak{n}$ basta quindi conoscere le relazioni moltiplicative che esprimono il prodotto di due elementi

$$
u+\mathfrak{n} \in K \cdot d_{i_{2}} K \ldots d_{i_{m}} K+\mathfrak{w} / \mathfrak{n}, \quad v+\mathfrak{n} \in K \cdot d_{j_{1}} K \ldots d_{j_{n}} K+\mathfrak{n} / \mathfrak{n}
$$

come elemento di $K \cdot d_{i_{1}} K \ldots d_{i_{m}} K \cdot d_{j_{1}} K \ldots d_{j_{n}} K+\mathfrak{n} / \mathfrak{n}$.

Ora, presciso dal caso in oui $i_{1}, \ldots, i_{m}, j_{1}, \ldots, j_{n}$ non siano $m+n$ indici distinti, in qual caso $u \cdot v+\mathfrak{n}$ sarebbe lo zero $\mathfrak{n}$, questo prodotto si riduce per mezzo di permutazioni $\pi, \pi^{\prime}$ aventi gli effetti

$$
\begin{gathered}
\pi\left(i_{1}\right)=1, \ldots, \pi\left(i_{m}\right)=m, \quad \pi\left(j_{1}\right)=m+1, \ldots, \pi\left(j_{n}\right)=m+n \\
\pi^{\prime}(m+1)=1, \pi^{\prime}(m+2)=2, \ldots, \pi^{\prime}(m+n)=n
\end{gathered}
$$

a $\pi(u \cdot v+\mathfrak{n})=\pi(u+\mathfrak{n}) \cdot \pi(v+\mathfrak{n})$, che ammette l'interpretazione dí un prodotto esterno $\pi(u+n) \wedge \pi^{\prime} \pi(v+\mathfrak{n})$.

In tale senso si può dire, che i calcoli nell' anello $M+\mathfrak{n} / \mathfrak{n} \cong M / 0$ danno una stretta concentrazione dei calcoli da eseguire nella corrispondenza differenziale $R / \mathfrak{n}$. (ved. 12).

355. I legami fra $R$ e $M$ e fra $R / \mathfrak{n}$ e $M+\mathfrak{n} / \mathfrak{n}$ mostrano che questi anelli esterni offrono già tutto quello, che il passaggio da un corpo $K$ di car $K \neq 2$ alle sue corrispondenze infinitesimale e differenziale può dare di essenziale allo studio della struttura di $K$. 
L'uguale importanza degli anelli $M$ e $M+\mathfrak{n} / \mathfrak{n} \cong M / \mathcal{d}$ e'induce a indicare con la sola sostituzione del segno $\equiv$ al segno ordinario di ugualità e cioè senza allusione al modulo $O$ della congruenza, il passaggio dall' anello $\mathrm{M}=[K, d K]$. che chiameremo semplicemente l'anello infinitesimale di $K$, all' anello $[K, d K] / 0$ da chiamarsi l'anello differenziale di $K$.

Mentre gli elementi di $[K, d K]$ si diranno infinitesimali $d i k$, di grado $n$ o $n$-pli se essi appartengono a $K \cdot(d K)^{n}$, estenderemo la denominazione differenziale di $K$ a tutti gli elementi dell'anello differenziale di $K$. Anche nel caso dei differenziali ̀ lecito di parlare del loro grado o della loro molteplicità, purchè essi appartengano $a$ un $K$-modulo $K \cdot(d K)^{n}+\mathfrak{n} / \mathfrak{n}$ senza essere $\equiv 0$. Infatti secondo 354 è diretta anche la decomposizione

$$
[K, d R] \equiv K+K \cdot d K+K \cdot d K \wedge d K+K \cdot d K \wedge d K \wedge d K+\ldots
$$

Per i gradi 0 e 1 non importano le distinzioni fra infinitesimali e differenziali e fra ugualità $=$ e congruenza $\equiv$. È più di una mera convenzione formale, se ammettiamo di considerare gli elementi di $K$ come differenziali di grado 0 .

356. L' importanza dell'anello differenziale sta nella possibilità di esten. dere l'operazione differenziazione, definita dapprima solamente per elementi di $K$, a tutti i differenziali.

'J' isomorfismo $\sigma_{i}$ di $K$, avente l'effetto $x^{\sigma_{i}}=x+d_{i} x(x \in K)$ può secondo 10 venire esteso a un automorfismo $\sigma_{i}$ dell' anello $R / \mathfrak{n}$, il che fornisce anche una estensione $d_{i}$ dell' operazione $d_{i}$, ponendo

$$
d_{i} X \equiv X^{\sigma_{i}}-X \quad \text { per ogni } X \in R / \mathfrak{n} .
$$

Di nuovo servirà l'anello differenziale. a una concisa descrizione di operazioni nella corrispondenza differenziale.

Per un differenziale $X$ di grado $n$ definiamo $d X \equiv d_{1}(\pi X)$ mediante una permutazione $\pi$ avente l'elfetto $\pi(i)=i+1$ per $i \leq n$, osservando, che questa definizione è d'accordo col senso $d_{1} X$ gia dato a $d X$ nel caso di $X \in K$ (ved. 349). Siccome ogni differenziale $X$ e somma di differenziali omogenei $X_{n}$ pienamente determinati da $X$ e dal loro grado $n$, possiamo definire $d X \equiv \Sigma d X_{n}$, chiamando questo il differenziale $d i X$. Sono evidenti allora le regole

$$
d(X+Y) \equiv d Y, \quad d(d X) \equiv 0 .
$$

Se $X \equiv a \cdot d x_{1} \wedge \ldots \wedge d x_{p}$ con $a \in K$, sarà $d X \equiv d a \wedge d x_{1} \wedge \ldots \wedge d x_{p}$, Il prodotto di $X$ con un simile differenziale $Y \equiv b \cdot d y_{1} \wedge \ldots \wedge d y_{q}$ ba quindi il differenziale $d(X \wedge Y) \equiv d(a \cdot b) \wedge d x_{1} \wedge \ldots \wedge d x_{p} \wedge d y_{1} \wedge \ldots \wedge d y_{q} \equiv d X \wedge Y+$ $+(-1)^{p} X \wedge d Y$, sicchè generalmente vale la regola

$$
d(X \wedge Y) \equiv d X \wedge Y+(-1)^{p} X \wedge d Y, \quad \text { purchè } X \text { sia di grado } p \text {. }
$$


35\%. In analogia alla definizione

$$
I_{n}(K)=\bigcap_{S} S \cdot(d S)^{n}, \quad \text { risp. } I_{n}\left(\frac{K}{k}\right)=\bigcap_{S \supset k} S \cdot(d S)^{n}
$$

dell'integrità infinitesimale di $K$ (sopra $k$ ) di grado $n$ (ved. 348), definiamo l'integrità differenziale $d i K$ (sopra $k$ ) di grado $n$ quale intersezione

$$
D_{n}(K)=\bigcap_{S}\left[S \cdot(d S)^{n}+\boldsymbol{O} / \mathrm{O}\right], \quad \text { risp. } D_{n}\left(\frac{K}{k}\right)=\bigcap_{S \supset k}\left[S \cdot(d S)^{n}+\mathrm{O} / \mathrm{O}\right],
$$

nella quale $S$ altresi percorre tutti gli aspetti perfetti di $K$ (contenenti $k$ ). Gli elementi di $D_{n}(K)\left(\right.$ di $\left.D_{n}\left(\frac{K}{k}\right)\right)$ saranno detti i differenziali integri n-pli di $K$ (sopra $k$ ).

Secondo le osservazioni fatte alla fine di $\mathbf{3 5 5}$ sarà lecito di identificare $I_{n}(K)=D_{n}(K), I_{n}\left(\frac{K}{k}\right)=D_{n}\left(\frac{K}{k}\right)$ per $n \leq 1$.

Valgono relazioni analoghe a quelle dimostrate in 349 :

$$
D_{p}(K) \wedge D_{q}(K) \subset D_{p+q}(K), \quad I_{0}(K) \cdot D_{n}(K) \subset D_{n}(K)
$$

(e. le altre riferentisi al caso relativo), alle quali deve aggiungersi la relazione

$$
d D_{n}(K) \subset D_{n+1}(K), \quad d D_{n}\left(\frac{K}{k}\right) \subset D_{n+1}\left(\frac{K}{k}\right)
$$

conseguenza immediata del fatto

$$
d(S+\mathrm{O} / \mathrm{O}) \subset d S+\mathrm{O} / \mathrm{O}
$$

All' anello graduato $I(K)=\sum_{n=0}^{\infty} I_{n}(K)$, risp. $I\left(\frac{K}{k}\right)=\sum_{n=0}^{\infty} I_{n}\left(\frac{K}{k}\right)$ (ved. 349), che chiameremo la totale integrità infinitesimale (sopra $k$ ), corrisponde la totale integrità differenziale di $K$ (sopra $k$ )

$$
D(K)=\sum_{n=0}^{\infty} D_{n}(K), \text { risp. } D\left(\frac{K}{k}\right)=\sum_{n=0}^{\infty} D_{n}\left(\frac{K}{k}\right),
$$

la quale è anello graduato ammettente differenziazione:

$$
d D(K) \subset D(K), d D\left(\frac{K}{k}\right) \subset D\left(\frac{K}{k}\right)
$$

(Conveniamo di riservare d'ora innanzi i simboli $I(K), I\left(\frac{K}{k}\right)$ alle totali integrità infinitesimali e di scrivere $I_{0}(K), I_{0}\left(\frac{K}{k}\right)$ per le ordinarie integrità prima (ved. 82) designate con quei simboli). 
358. Da $\left[\bigcap_{S} S \cdot(d S)^{n}\right]+\partial \subset \bigcap_{s}\left[S \cdot(d S)^{n}+0\right]$ segue che ogni infinitesimale integro $X$ di grado $n$ individua un differenziale integro congruo a quello, sicchè si ottiene un omomorfismo dell'anello $I(K)\left(\right.$ di $\left.I\left(\frac{K}{k}\right)\right)$ nell' anello $D(K)$ (in $D\left(\frac{K}{k}\right)$ ). In riguardo a questo legame tra differenziali e infinitesimali integri sono importanti gli infinitesimali antisimmetrici.

$L$ 'antisimmetria di un infinitesimale

$$
X=\Sigma a_{i_{1} \ldots i_{n}} \cdot d x_{i_{1}} \wedge \ldots \wedge d x_{i_{n}}=\Sigma a_{i_{1} \ldots i_{n}} \cdot d_{1} x_{i_{1}} \ldots d_{n} x_{i_{n}}, \quad\left(x_{i} \in B\right),
$$

espresso mediante una base $B$ del tipo descritto in 351, significa

$$
\pi^{-1} X=\Sigma a_{i_{1} \ldots i_{n}} \cdot d_{\left.\pi_{(1)}^{-1}\right)} x_{i_{1}} \ldots d_{\pi_{(n)}^{-1} x_{i_{n}}}=\chi\left(\pi^{-1}\right) \cdot X \quad \text { (ved. 350) }
$$

ed equivale pertanto all' antisimmetria

$$
a_{i_{1} \ldots i_{n}}=\chi(\pi) \cdot a_{i_{\pi(1)} \ldots i_{\pi(n)}}
$$

del *tensore» $a_{i_{1} \ldots i_{n}}$, data l'indipendenza lineare dei prodotti $d_{1} x_{i_{1}} \ldots a_{n} x_{i_{n}}$ (ved. 351). Ponendo quindi il determinante $\Sigma_{\pi} \chi(\pi) \cdot d x_{i_{\pi(1)}} \wedge \cdots \wedge d x_{i_{\pi(n)}}=$ $=\Sigma \chi(\pi) \cdot d_{1} x_{i_{\pi(1)}} \ldots d_{n} x_{i_{\pi(n)}}$ uguale a $d\left(x_{i_{1}}, \ldots, x_{i_{n}}\right)^{\pi}$, potremo contrarre l'espres. sione di $X$ a

$$
X=\Sigma^{\prime} a_{i_{1} \ldots i_{n}} \cdot d\left(x_{i_{1}}, \ldots, x_{i_{n}}\right),
$$

ove $\Sigma^{\prime}$ significa sommazione sotto le condizioni: $i_{1}<\ldots<i_{n}$.

Dato un differenziale $n$-plo

$$
X \equiv \underset{i_{1} \ldots i_{n}}{\Sigma^{\prime}} a_{i_{1} \ldots i_{n}} \cdot d x_{i_{1}} \wedge \ldots \wedge d x_{i_{n}},
$$

ridotto, come indica il segno $\Sigma^{\prime}$ (red. 353), gli associamo l'infinitesimale antisimmetrico

$$
Y=\underset{i_{1} \ldots i_{n}}{\Sigma^{\prime}} a_{i_{1} \ldots i_{n}} \cdot d\left(x_{i_{1}}, \ldots, x_{i_{n}}\right)
$$

che di seguito a $X \equiv \chi(\pi) . \underset{i_{1} \ldots i_{n}}{\Sigma^{\prime}} a_{i_{1} \ldots i_{n}} \cdot d x_{i_{\pi(1)}} \wedge \ldots \wedge d x_{i_{\pi(n)}}$ è congruo a $n ! X$. Non interessa quindi il caso di car $K=2$, qualora non sia $n=1$.

Dimostreremo che, presciso dal caso di car $K=2, n>1$, quell'infinitesimale $Y$ è integro (sopra $k$ ), purchè $X$ sia integro (sopra $k$ ).

Sia $S$ aspetto perfetto (contenente $k$ ) qualunque e

$$
X \equiv \Sigma e \cdot d f_{1} \wedge \cdots \wedge d f_{n} \quad\left(e, f_{1}, \ldots, f_{n} \in S\right) .
$$

Introducendo le espressioni $d f=\sum_{i} \frac{\partial f}{\partial x_{i}} \cdot d x_{i}$ di $d f_{1}$, ecc. mediante la base $B$, otteniamo

$X \equiv \Sigma \underset{i_{1} \ldots i_{n}}{\Sigma} e \cdot \frac{\partial f_{1}}{\partial x_{i_{1}}} \ldots \frac{\partial f_{n}}{\partial x_{i_{n}}} \cdot d x_{i_{1}} \wedge \ldots \wedge d x_{i_{n}} \equiv \Sigma \underset{i_{1} \ldots i_{n}}{\Sigma} e \cdot \frac{\partial\left(f_{1}, \ldots, f_{n}\right)}{\partial\left(x_{i_{1}}, \ldots, x_{i_{n}}\right)} \cdot d x_{i_{2}} \wedge \ldots \wedge d x_{i_{n}}$ 
e dal confronto di questa espressione col membro destro di (*) risultano le equazioni

$$
a_{i_{1} \ldots i_{n}}=\Sigma e \cdot \frac{\partial\left(f_{1}, \ldots, f_{n}\right)}{\partial\left(x_{i_{1}}, \ldots, x_{i_{n}}\right)},
$$

giacchè un infinitesimale della forma ridotta non può appartenere a $\partial$ che quando esso è 0 (ved. 3522 o 353). Quelle espressioni per i coefficienti permettono di scrivere $Y$ nella forma

$$
\begin{gathered}
Y=\Sigma \Sigma_{i_{1} \ldots i_{n}}^{\Sigma^{\prime}} e \cdot \frac{\partial\left(f_{1}, \ldots, f_{n}\right)}{\partial\left(x_{i_{1}}, \ldots, x_{i_{n}}\right)} \cdot d\left(x_{i_{1}}, \ldots, x_{i_{n}}\right)= \\
=\Sigma \underset{\pi i_{1} \ldots i_{n}}{\Sigma} e_{i}^{\prime} \cdot \frac{\partial\left(f_{1}, \ldots, f_{n}\right)}{\partial\left(x_{i_{1}}, \ldots, x_{i_{n}}\right)} \cdot \chi(\pi) \cdot d_{1} x_{i_{\pi(1)}} \ldots d_{n} x_{i_{\pi(n)}}= \\
=\Sigma \underset{i_{1} \ldots i_{n}}{\Sigma} e \cdot \frac{\partial\left(f_{1}, \ldots, f_{n}\right)}{\partial\left(x_{i_{1}}, \ldots, x_{i_{n}}\right)} \cdot d_{1} x_{i_{1}} \ldots d_{n} x_{i_{n}}=\Sigma \underset{\pi}{\Sigma} \chi(\pi) \cdot e \cdot d f_{\pi(1)} \wedge \ldots \wedge d f_{\pi(n)}
\end{gathered}
$$

il che dimostra $Y \subset S \cdot(d S)^{n}$ e quindi l'integrità di $Y$.

Pur non potendo affermare che ogni differenziale integro sia congruo a un infinitesimale integro, siamo tuttavia riusciti a provare, che $l \cdot n !-p l o$ di ogni differenziale n-plo integro (sopra $k$ ) $\dot{e}$ congruo a un infinitesimale antisimmetrico integro (sopra $k$ ).

\section{\& 4. Caleole dolle integrità di nn corpe.}

359. La determinazione delle integrità di un corpo $K$ si facilita, se $K$ viene considerato come sopra-corpo finito e separabile di un corpo abbastanza semplice $K_{0}$.

Rammentiamo, che ogni aspetto perfetto di $K$ determina un aspetto $S_{0}=S \frown K_{0}$ di $K_{0}$ (ved. 328) che nel caso presente, dove $\operatorname{dim} \frac{K}{K_{0}}=0$, non è totale (ved. 126) e pertanto perfetto. Del resto $\mathbb{P}_{0}$ è l'unica prospettiva perfetta di $K_{0}$, della quale $\mathbb{D}$ sia estensione, perchè ogni tale aspetto è contenuto in $S \cap K_{0}$ e quindi uguale a $S \cap K_{0}$ (ved. 96).

La separabilità di $K$ sopra $K_{0}$ induce $\partial_{0}\left(\frac{S}{S_{0}}\right) \neq 0$ (ved. 285), il che rende utile il lemma che segue:

360. Se l'infinitesimale $n-p l o x$ soddisfa alla condizione

$$
x \subset S \cdot(d S)^{n}
$$

e l'aspetto perfetto $S$ ha base algebrica sopra $S_{0} \leftarrow S$, allora vale

$$
x \cdot \partial_{0}\left(\frac{S}{S_{0}}\right)^{n} \subset \partial_{1}\left(\frac{S}{S_{0}}\right)^{n} \cdot\left(d S_{0}\right)^{n}
$$

